\title{
On the factors of the jacobian variety of a modular function field
}

\author{
By Goro SHIMURA
}

(Received Nov. 13, 1972)

\section{$\S 0$. Introduction.}

For a positive integer $N$, put

$$
\begin{aligned}
& \Gamma_{0}(N)=\left\{\left[\begin{array}{ll}
a & b \\
c & d
\end{array}\right] \in S L_{2}(\boldsymbol{Z}) \mid c \equiv 0(\bmod N)\right\}, \\
& \Gamma_{1}(N)=\left\{\left[\begin{array}{ll}
a & b \\
c & d
\end{array}\right] \in \Gamma_{0}(N) \mid a \equiv d \equiv 1(\bmod N)\right\} .
\end{aligned}
$$

We consider any group $\Gamma$ such that $\Gamma_{1}(N) \subset \Gamma \subset \Gamma_{0}(N)$, and call it a group of level $N$. Let $J$ denote the jacobian variety of the compact Riemann surface $\mathfrak{S}^{*} / \Gamma$, where $\mathfrak{S}^{*}$ means the union of the upper half plane

$$
\mathscr{S}=\{z \in C \mid \operatorname{Im}(z)>0\}
$$

and the cusps of $\Gamma$. Further let $S_{k}(\Gamma)$ be the vector space of all holomorphic cusp forms of weight $k$ with respect to $\Gamma$. Then, with each common eigenfunction $f(z)$ in $S_{2}(\Gamma)$ of the Hecke operators $T_{n}$ for all $n$, one can associate an abelian variety $A$ that is a "factor" of $J$. The purpose of this note is to consider a few arithmetical questions concerning the correspondence between $f$ and $A$. Besides, as an application of our methods, we shall give a proof of Dirichlet's class number formula for an imaginary quadratic field, without using the residue technique.

We start our treatment by proving that $A$ can naturally be obtained as a quotient of $J$ by an abelian subvariety rational over $\boldsymbol{Q}$ (Theorem 1 ). Actually in $[11, \S 7.5]$, we gave a formulation with such a factor as a subvariety of $J$. The two formulations are essentially equivalent, but there is a subtle difference. At any rate, they are connected by the following fact: there is a canonical $\boldsymbol{C}$-linear isomorphism of $S_{2}(\Gamma)$ onto the tangent space of $J$ at the origin, which has a certain commutative property with the action of Hecke operators. Such an isomorphism was used in the proofs of [11, Th. 7.14, Prop. 7.19] and also in [12], but not explicitly given. This point will be clarified in $\S 2$. It will be shown in $\S 3$ that $A$ can be obtained as a complex torus whose periods are those of $f$ and some other cusp forms. We shall consider 
in $\S 4$ how the map

$$
f(z)=\sum_{n=1}^{\infty} a_{n} e^{2 \pi i n z} \longmapsto \sum_{n=1}^{\infty} \chi(n) a_{n} e^{2 \pi i n z}
$$

with a numerical character $\chi$, can be reflected by geometric objects. If $\chi$ is a character of order 2, it corresponds to a homomorphism of $A$ rational over the quadratic field associated with $\chi$. Under certain conditions, $A$ is similar to abelian varieties of the type discussed in $[13, \S 9]$. Therefore the coordinates of some specific points of finite order on such an $A$ can generate

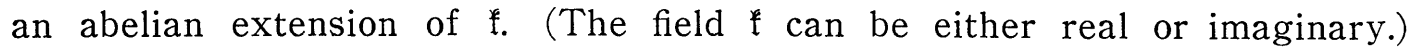
Recently K. Doi and M. Yamauchi have found some interesting arithmetical relations for the Fourier coefficients of certain eigen-functions in $S_{2}\left(\Gamma_{0}\left(p^{3}\right)\right)$ with a prime $p$. It is expected that these will be understood in the framework of $[13, \S 9]$, under the formulation of $\S 4$ of the present paper.

The next $\S 5$ is devoted to a proof of Dirichlet's formula for the class number $h(-q)$ of an imaginary quadratic field $\boldsymbol{Q}(\sqrt{-q})$ of discriminant $-q$. In the previous papers [12], [13], we showed that $A$ has complex multiplication if and only if the Mellin transform of $f$ is an $L$-function with a Grössencharacter of an imaginary quadratic field. When the field is $\boldsymbol{Q}(\sqrt{-q})$, the number of such characters, under certain conditions, is $h(-q)$ times a simple factor. Combining these facts with some other observations about eigenfunctions, we find a relation between $h(-q)$ and the trace of the map $\left(^{*}\right)$ on $S_{2}(\Gamma)$ for a certain $\Gamma$ Proposition 10. Then Dirichlet's formula can be obtained by computing the trace by the Riemann-Roch theorem or by the Selberg-Eichler trace formula. The whole idea is under the influence of Hecke's works [4], [5], in which Hecke computed the multiplicities of the irreducible representations of $P S L_{2}(\boldsymbol{Z} / q \boldsymbol{Z})$ in a certain space of cusp forms, when $q$ is a prime. However, here we need no information about such representations. Although the proof is by no means simple nor elementary, yet the author thinks that this method is natural and opens some possibilities of generalization.

In the final $\S 6$, we shall briefly explain a method of determining the zetafunction of $A$ over a certain type of non-abelian extension of $\boldsymbol{Q}$.

\section{$\S 1$. The factor $A$ as a quotient of $J$.}

Let us start with some definitions and notational convention. For an abelian variety $B$ defined over $C$, we denote by $D_{B}$, or $D(B)$, the vector space over $C$ of all holomorphic 1 -forms on $B$, and by $Y_{B}$, or $Y(B)$, the tangent space of $B$ at the origin. Then $D_{B}$ is dual to $Y_{B}$, so that we have a $C$ bilinear pairing 


$$
(,)_{B}: D_{B} \times Y_{B} \longrightarrow C \text {. }
$$

If $\alpha$ is a homomorphism of $B$ to another abelian variety $B^{\prime}$, one can naturally define two maps

which satisfy

$$
\begin{array}{ll}
d \alpha: & Y_{B} \longrightarrow Y_{B^{\prime}}, \\
\delta \alpha: & D_{B^{\prime}} \longrightarrow D_{B},
\end{array}
$$

$$
(\delta \alpha u, v)_{B}=(u, d \alpha v)_{B^{\prime}} \quad\left(u \in D_{B^{\prime}}, v \in Y_{B}\right) .
$$

By a quotient of $B$ by an abelian subvariety, say $C$, of $B$, we understand a couple $(A, \nu)$ formed by an abelian variety $A$ which canonically represents $B / C$ and a natural map $\nu: B \rightarrow A$ with $C=\operatorname{Ker}(\nu)$ (cf. Chow [3]). We see easily that

$$
Y_{C}=\left\{v \in Y_{B} \mid(u, v)_{B}=0 \text { for all } u \in \delta \nu\left(D_{A}\right)\right\} .
$$

Let $\Gamma$ be a group of level $N$, and $J$ the jacobian variety of $\mathscr{S}^{*} / \Gamma$. Let $\psi: \mathfrak{S}^{*} \rightarrow J$ be the map obtained by composing the natural map $\mathfrak{S}^{*} \rightarrow \mathfrak{S}^{*} / \Gamma$ with a canonical map of $\mathscr{S}^{*} / \Gamma$ into $J$. Then there is an isomorphism $\mu: S_{2}(\Gamma) \rightarrow D_{J}$ defined by $\delta \psi(\mu(f))=f(z) d z$ for $f \in S_{2}(\Gamma)$, where $\delta \phi$ is the "pull back" associated with $\phi$, and $z$ is a standard variable on $\mathfrak{H}$.

Let $\Gamma^{\prime}$ be a group of level $M$, and let

$$
\tilde{\Gamma}=\left\{\alpha \in \boldsymbol{R} \cdot G L_{2}(\boldsymbol{Q}) \mid \operatorname{det}(\alpha)>0\right\} .
$$

Define, for $\alpha \in \tilde{\Gamma}$, an algebraic correspondence $X\left(\Gamma^{\prime} \alpha \Gamma\right)$ on $\mathfrak{S}^{*} / \Gamma \times \mathscr{S}^{*} / \Gamma^{\prime}$ as in $[11, \S 7.2]$. (Briefly, it is the locus of $z \times \alpha(z)$ modulo $\Gamma \times \Gamma^{\prime}$.) Let $J^{\prime}$ and $\mu^{\prime}$ denote the corresponding geometric objects defined with $\Gamma^{\prime}$ in place of $\Gamma$. One can attach to $X\left(\Gamma^{\prime} \alpha \Gamma\right)$ a homomorphism of $J$ into $J^{\prime}$ as in Weil [15, $\left.\S \mathrm{VI}\right]$, which we denote by $\left\{\Gamma^{\prime} \alpha \Gamma\right\}$. Now the commutative diagrams (7.2.2) and (7.2.6) of [11] imply

$$
\mu \circ\left[\Gamma^{\prime} \alpha \Gamma\right]_{2}=\delta\left\{\Gamma^{\prime} \alpha \Gamma\right\} \circ \mu^{\prime},
$$

where $\left[\Gamma^{\prime} \alpha \Gamma\right]_{k}$ is the map $S_{k}\left(\Gamma^{\prime}\right) \rightarrow S_{k}(\Gamma)$ defined in $[11, \S 3.4]$. Then, from (1.1) and (1.3), we obtain

$$
\left(\mu^{\prime}(f), d\left\{\Gamma^{\prime} \alpha \Gamma\right\} v\right)_{J^{\prime}}=\left(\mu\left(f \mid\left[\Gamma^{\prime} \alpha \Gamma\right]_{2}\right), v\right)_{J} \quad\left(f \in S_{2}\left(\Gamma^{\prime}\right), v \in Y_{J}\right) .
$$

For a positive integer $n$, let $q$ be the largest divisor of $n$ prime to $N$, and put $n=m q$. Then we define $T_{n}$ and $\xi_{n}$ by $T_{n}=T_{m} T_{q}, \xi_{n}=\xi_{m} \xi_{q}, T_{m}=[\Gamma \alpha \Gamma]_{2}$, $\xi_{m}=\{\Gamma \alpha \Gamma\}$ with $\alpha=\left[\begin{array}{rr}1 & 0 \\ 0 & m\end{array}\right]$, and $T_{q}=\sum_{\beta}[\Gamma \beta \Gamma]_{2}, \quad \xi_{q}=\sum_{\beta}\{\Gamma \beta \Gamma\}$, where $\sum_{\beta}$ is extended over all distinct $\Gamma \beta \Gamma$ with $\operatorname{det}(\beta)=q, \beta \equiv\left[\begin{array}{ll}1 & 0 \\ 0 & q\end{array}\right](\bmod N)$. Then $\mu \circ T_{n}=\delta \xi_{n} \circ \mu$. 
As shown in [11, §7.3], there is a "standard model" of $\mathfrak{S}^{*} / \Gamma$ defined over $\boldsymbol{Q}$ (see also $\S 4$ below), so that $J$ can naturally be defined over $\boldsymbol{Q}$. Let $f(z)=\sum_{n=1}^{\infty} a_{n} e^{2 \pi i n z}$, with $a_{1}=1$, be an element of $S_{2}(\Gamma)$, that is a common eigenfunction of $T_{n}$ for all $n$ (i. e., $f \mid T_{n}=a_{n} f$ ). Fix $f$, and let $K$ be the subfield of $\boldsymbol{C}$ generated over $\boldsymbol{Q}$ by the complex numbers $a_{n}$ for all $n$. (Of course $K$ depends on $f$.) Then we have

THEOREM 1. There exists a triple $(A, \nu, \theta)$ formed by the objects satisfying the following conditions:

(i) $(A, \nu)$ is a quotient of $J$ by an abelian subvariety rational over $\boldsymbol{Q}$.

(ii) $\theta$ is an isomorphism of $K$ into End $(A) \otimes \boldsymbol{Q}$ such that $\nu \circ \xi_{n}=\theta\left(a_{n}\right) \circ \nu$ for all $n$. (This implies especially $\theta(1)=i d_{A}$.)

(iii) $\operatorname{dim}(A)=[K: \boldsymbol{Q}]$.

Moreover, let $I$ denote the set of all isomorphisms of $K$ into $C$. Then, for every $\sigma \in I$, there exists an element $f_{\sigma}$ of $S_{2}(\Gamma)$ such that $f_{\sigma} \mid T_{n}=a_{n}^{\sigma} f$ for all $n$, and $f_{\sigma}(z)=\sum_{n=1}^{\infty} a_{n}^{\sigma} e^{2 \pi i n z}$. With these $f_{\sigma}$, one has

(iv) $\delta \nu\left(D_{A}\right)=\mu\left(\sum_{o \in I} \boldsymbol{C} f_{\sigma}\right)$.

Under the conditions (i, ii), the triple $(A, \nu, \theta)$ is unique up to isomorphisms over $\boldsymbol{Q}$. Furthermore, the one-dimensional part of the zeta function of $A$ over $\boldsymbol{Q}$ coincides, up to finitely many Euler factors for bad primes, with $\prod_{\sigma \in I}\left(\sum_{n=1}^{\infty} a_{n}^{\sigma} n^{-s}\right)$, provided that $\Gamma=\Gamma_{0}(N)$, or $\sum_{\sigma \in I} \boldsymbol{C} f_{\sigma}$ is stable under the map $h(z) \mapsto h(-1 / N z) / z^{2}$.

Proof. Let $\mathfrak{I}$ be the subalgebra of End $(J) \otimes \boldsymbol{Q}$ generated by the $\xi_{n}$ for all $n$, and $\mathfrak{R}$ the radical of $\mathfrak{I}$. Then $\mathfrak{I}=\Re \oplus \mathfrak{S}$ with a commutative semisimple algebra $\subseteq$, whose simple components we denote by $\Re_{1}, \cdots, \Re_{r}$. By (1.3), we see that $\delta \xi_{n}$ maps $\mu(f)$ onto $a_{n} \cdot \mu(f)$, hence we can define a homomorphism $\rho$ of $\mathfrak{I}$ onto $K$ by $\rho\left(\xi_{n}\right)=a_{n}$. We may assume, changing the order if necessary, that $\rho$ gives an isomorphism of $\Re_{1}$ onto $K$. Let $\rho^{\prime}: K \rightarrow \Re_{1}$ denote its inverse map, and put $\mathfrak{U}=\Re_{2}+\cdots+\Re_{r}+\Re$. Let $C$ be the abelian subvariety of $J$ generated by $\alpha(J)$ for all $\alpha \in \mathfrak{U} \cap \operatorname{End}(J)$. Since the elements of $\mathfrak{I} \cap$ End $(J)$ are rational over $\boldsymbol{Q}$, we can construct a quotient $(A, \nu)$ of $J$ by $C$ rational over $\boldsymbol{Q}$. Observe that $\mathfrak{U}$ is an ideal of $\mathfrak{I}$, hence $C$ is stable under $\mathfrak{R}_{1} \cap$ End $(J)$. Therefore we can define a homomorphism $\theta: K \rightarrow$ End $(A) \otimes \boldsymbol{Q}$ such that $\nu \circ \rho^{\prime}(a)=\theta(a) \circ \nu$ for all $a \in K$. Since $\xi_{n}-\rho^{\prime}\left(a_{n}\right) \in \mathfrak{H}$, we obtain $\nu \circ \xi_{n}=\theta\left(a_{n}\right) \circ \nu$ for all $n$. To prove $J \neq C$, put $\mathfrak{I}_{C}=\mathfrak{I} \otimes_{Q} C$. By [11, Th. 3.51], $S_{2}(\Gamma)$ is isomorphic to $\mathfrak{I}_{C}$ as a $\mathfrak{I}_{C}$-module, hence $D_{J}$ is (via $\delta$ ) $\mathfrak{I}_{C}$-isomorphic to $\mathfrak{I}_{C}$. If $J=C$, we have

$$
\{0\}=\left\{x \in D_{J} \mid \delta \alpha(x)=0 \text { for all } \alpha \in \mathfrak{H}\right\},
$$

so that the $\mathfrak{I}_{C}$-isomorphism between $D_{J}$ and $\mathfrak{I}_{C}$ implies

$$
\{0\}=\left\{\xi \in \mathfrak{I}_{\boldsymbol{c}} \mid \mathfrak{H} \xi=0\right\} .
$$


Take the largest non-negative integer $q$ such that $\Re_{1} \Re^{q} \neq\{0\}$. (We understand that $\mathfrak{R}^{0}=\mathfrak{I}$.) Then the right hand side of $\left(^{*}\right)$ contains $\Re_{1} \Re^{q}$, a contradiction. Thus $J \neq C$, so that $\operatorname{dim}(A)>0$, and $\theta$ is injective. Now suppose that $\left(A^{\prime}, \nu^{\prime}, \theta^{\prime}\right)$ satisfies (i, ii). Then the elements of $\theta^{\prime}(K) \cap$ End $\left(A^{\prime}\right)$ are rational over $\boldsymbol{Q}$, hence the representation of $K$ on $D_{A^{\prime}}$ via $\theta^{\prime}$ is a multiple of a regular representation of $K$ over $\boldsymbol{Q}$. Therefore, if $m$ is the multiplicity, $D_{A^{\prime}}$ has a basis $\left\{w_{\sigma j} \mid \sigma \in I, 1 \leqq j \leqq m\right\}$ over $\boldsymbol{C}$ such that $\delta \theta^{\prime}(a)\left(w_{\sigma j}\right)=a^{\sigma} w_{\sigma j}$ for all $a \in K$. Let $f_{\sigma j}=\mu^{-1}\left(\delta \nu^{\prime}\left(w_{\sigma j}\right)\right)$. Then $f_{\sigma j} \mid T_{n}=a_{n}^{\sigma} f_{\sigma j}$ for all $n$. It follows that $m=1$, hence $\operatorname{dim}\left(A^{\prime}\right)=[K: Q]$. This shows also the existence of $f_{\sigma}$ as stated in our theorem, and $\delta \nu^{\prime}\left(D_{A^{\prime}}\right)=\mu\left(\sum_{\sigma \in I} C f_{\sigma}\right)$. By (1.2), the tangent space of $\operatorname{Ker}\left(\nu^{\prime}\right)$ at the origin is the annihilator of $\mu\left(\sum_{\sigma \in I} \boldsymbol{C} f_{\sigma}\right)$. This proves the uniqueness of $(A, \nu, \theta)$ and the property (iv). The assertion concerning the zeta-function of $A$ can easily be proved by shifting the congruence relation $[11,(7.5 .1,2)]$ to $A$.

As to the zeta-function, we have somewhat more generally

Proposition 1. Suppose $\Gamma=\Gamma_{0}(N)$. Let $(A, \nu)$ be a quotient of $J$ by an abelian subvariety rational over $\boldsymbol{Q}$. Then $\mu^{-1}\left(\delta \nu\left(D_{A}\right)\right)$ is stable under $T_{n}$ for all $n$ prime to $N$. Moreover, if $T_{n}^{\prime}$ denotes the restriction of $T_{n}$ to $\mu^{-1}\left(\delta \nu\left(D_{A}\right)\right)$, then the one-dimensional part of the zeta-function of $A$ over $\boldsymbol{Q}$ coincides, $u p$ to finitely many Euler factors, with $\operatorname{det}\left(\sum_{(n, N)=1} T_{n}^{\prime} n^{-s}\right)$.

Proof. By [11, Prop. 7.19], we see that $\operatorname{Ker}(\nu)$ is stable under $\xi_{n}$ for all $n$ prime to $N$. Then (1.2) shows that $\delta \nu\left(D_{A}\right)$ is stable under $\delta \xi_{n}$ for all such $n$, hence the first assertion. Also, we can define an endomorphism $\xi_{n}^{\prime}$, for such an $n$, of $A$ such that $\xi_{n}^{\prime} \circ \nu=\nu \circ \xi_{n}$. Shifting the congruence relation [11, (7.5.1)] to $A$, we obtain the second assertion.

\section{§2. The factor $A_{0}$ as a subvariety of $J$ and a canonical isomorphism of $S_{2}(\Gamma)$ onto $Y_{J}$.}

Let $\alpha=\left[\begin{array}{ll}a & b \\ c & d\end{array}\right] \in G L_{2}(\boldsymbol{R}), \operatorname{det}(\alpha)>0$. For a complex valued function $f(z)$ on $\mathfrak{S}$ and a positive integer $k$, we define a function $f \mid[\alpha]_{k}$ on $\mathfrak{S}$ by

$$
\left(f \mid[\alpha]_{k}\right)(z)=\operatorname{det}(\alpha)^{k / 2}(c z+d)^{-k} f(\alpha(z)),
$$

where $\alpha(z)=(a z+b) /(c z+d)$ for $z \in \mathfrak{H}_{3}$. Put $\omega_{N}=N^{-1 / 2}\left[\begin{array}{ll}0 & 1 \\ N & 0\end{array}\right]$, and define a function $f \mid\left[\omega_{N}\right]_{k}$ on $\mathscr{S}$ by

$$
\left(f \mid\left[\omega_{N}\right]_{k}\right)(z)=N^{-k / 2} z^{-k} \bar{f}(1 / N \bar{z}),
$$

where bars mean the complex conjugation. Then one can easily verify:

$$
\left(f \mid[\alpha]_{k}\right)\left|\left[\omega_{N}\right]_{k}=\left(f \mid\left[\omega_{M}\right]_{k}\right)\right|\left[\omega_{M}^{-1} \alpha \omega_{N}\right]_{k},
$$




$$
\begin{aligned}
& f \mid\left[\omega_{N}\right]_{k}^{2}=f, \\
& \omega_{N}^{-1} \Gamma \omega_{N}=\Gamma \text { for any group } \Gamma \text { of level } N .
\end{aligned}
$$

It follows that $\left[\omega_{N}\right]_{k}$ induces an anti- $C$-linear automorphism of $S_{k}(\Gamma)$. Moreover, let $\Gamma^{\prime}$ be a group of level $M$, and let $\alpha \in \tilde{\Gamma}$. Then

$$
\left(f \mid\left[\Gamma^{\prime} \alpha \Gamma\right]_{k}\right)\left|\left[\omega_{N}\right]_{k}=\left(f \mid\left[\omega_{M}\right]_{k}\right)\right|\left[\Gamma^{\prime} \omega_{M}^{-1} \alpha \omega_{N} \Gamma\right]_{k} \quad\left(f \in S_{k}\left(\Gamma^{\prime}\right)\right) .
$$

If $\langle$,$\rangle denotes the Petersson inner product on S_{k}(\Gamma)$, then

$$
\left\langle f, g \mid\left[\omega_{N}\right]_{k}\right\rangle=\left\langle g, f \mid\left[\omega_{N}\right]_{k}\right\rangle .
$$

Let $\mu: S_{2}(\Gamma) \rightarrow D_{J}$ be the map defined in $\S 1$. We can now define a $\boldsymbol{C}$ linear isomorphism $\lambda: S_{2}(\Gamma) \rightarrow Y_{J}$ by

$$
(\mu(f), \lambda(g))_{J}=i \cdot\left\langle f, g \mid\left[\omega_{N}\right]_{2}\right\rangle \quad\left(f, g \in S_{2}(\Gamma)\right) .
$$

The constant factor $i$ is not absolutely necessary, but will make a later discussion smooth. Put $Y^{\prime}=Y\left(J^{\prime}\right)$ with the jacobian variety $J^{\prime}$ of $\mathfrak{S}^{*} / \Gamma^{\prime}$.

Define $\lambda^{\prime}: S_{2}\left(\Gamma^{\prime}\right) \rightarrow Y^{\prime}$ in the same manner. Combining (2.4) and (1.4) with $[11,(3.4 .5)]$, we obtain a relation

$$
\lambda\left(f \mid\left[\Gamma^{\prime} \alpha \Gamma\right]_{2}\right)=d\left\{\Gamma \omega_{N}^{-1} \alpha^{\prime} \omega_{M} \Gamma^{\prime}\right\}\left(\lambda^{\prime}(f)\right) \quad \text { for } \quad f \in S_{2}\left(\Gamma^{\prime}\right),
$$

where $\iota$ denotes the main involution, i. e., $\left[\begin{array}{ll}a & b \\ c & d\end{array}\right]^{c}=\left[\begin{array}{rr}d & -b \\ -c & a\end{array}\right]$.

Consider the special case in which $M=N, \Gamma^{\prime}=\Gamma$, and $\alpha=\left[\begin{array}{ll}1 & 0 \\ 0 & n\end{array}\right]$ with a positive integer $n$, or $\alpha \equiv\left[\begin{array}{ll}1 & 0 \\ 0 & q\end{array}\right](\bmod N)$, $\operatorname{det}(\alpha)=q,(q, N)=1$. Then $[2.7)$ implies

$$
\lambda\left(f \mid T_{n}\right)=d \xi_{n}(\lambda(f)) \quad \text { for } \quad f \in S_{2}(\Gamma) .
$$

Further let $q$ be an integer prime to $N, \sigma_{q}$ an element of $S L_{2}(\boldsymbol{Z})$ such that $q \cdot \sigma_{q} \equiv\left[\begin{array}{ll}1 & 0 \\ 0 & q^{2}\end{array}\right](\bmod N)$, and let $\tau_{N}=\left[\begin{array}{cr}0 & -1 \\ N & 0\end{array}\right]$. Then we obtain from $(2.7)$ the following relations :

$$
\begin{array}{ll}
\lambda\left(f \mid\left[\sigma_{q}\right]_{2}\right)=d\left\{\Gamma \sigma_{q} \Gamma\right\}(\lambda(f)) & \text { for } \quad f \in S_{2}(\Gamma), \\
\lambda\left(f \mid\left[\tau_{N}\right]_{2}\right)=d\left\{\Gamma \tau_{N} \Gamma\right\}(\lambda(f)) & \text { for } \quad f \in S_{2}(\Gamma) .
\end{array}
$$

The formula (2.7) and its specializations (2.8-10) are the commutative property of the isomorphism $\lambda$ mentioned in the Introduction.

Now define $K$ for a common eigen-function $f(z)=\sum_{n=1}^{\infty} a_{n} e^{2 \pi i n z}$, with $a_{1}=1$, as in $\S 1$. Then [11, Th. 7.16] can be re-stated as follows.

THEOREM 2. There exists an abelian subvariety $A_{0}$ of $J$ and an isomorphism $\theta_{0}$ of $K$ into End $\left(A_{0}\right) \otimes \boldsymbol{Q}$ with the following properties: 
(i) $\operatorname{dim}\left(A_{0}\right)=[K: Q]$;

(ii) $\theta_{0}\left(a_{n}\right)$ is the restriction of $\xi_{n}$ to $A_{0}$ for all $n$;

(iii) $A_{0}$ is rational over $\boldsymbol{Q}$.

(iv) If $f_{\sigma}$ for $\sigma \in I$ is defined as in Theorem 1, then $Y\left(A_{0}\right)=\lambda\left(\sum_{\sigma \in I} \boldsymbol{C} f_{\sigma}\right)$. Moreover the couple $\left(A_{0}, \theta_{0}\right)$ is unique under (i) and (ii).

The last property (iv) of $A_{0}$ is not explicitly stated in [11, Th. 7.16], but follows immediately from its proof.

The formulation of the results of [12] and their proofs require the isomorphism $\lambda$. Let us now clarify a few points in [12], where the explanation may be somewhat vague. In the proof of $[12, \mathrm{Th} .1]$, we should put $\delta=$ $\left[\begin{array}{cc}1 & 1 / D \\ 0 & 1\end{array}\right]$, which is erroneously printed as $\left[\begin{array}{cc}1 & 1 / d \\ 0 & 1\end{array}\right]$. Then the endomorphism $\omega$ of $A^{\prime}$ mentioned there is $\{\Gamma \varepsilon \Gamma\}$ with $\varepsilon=\left[\begin{array}{cc}1 & 0 \\ -M / D & 1\end{array}\right]$. Similarly the homomorphism $\xi^{*}, \phi$, and $\varphi$ are given by $\xi^{*}=\sum_{u} t x_{u} \cdot\left\{\Gamma_{1}\left(M^{\prime}\right) \omega_{M^{\prime}}^{-1} \eta_{u}^{t} \omega_{M} \Gamma_{1}(M)\right\}$, $\phi=\left\{\Gamma_{1}(M) \omega_{M}^{-1} \beta^{c} \omega_{L} \Gamma_{1}(L)\right\}, \varphi=\left\{\Gamma_{1}(M) \omega_{M}^{-1} \omega_{L} \Gamma_{1}(L)\right\}$. One can also formulate the results of [12] in terms of $(A, \nu, \theta)$ of Theorem 1 .

Proposition 2. Let $(A, \nu, \theta)$ be as in Theorem 1 , and $\left(A_{0}, \theta_{0}\right)$ be as in Theorem 2. Suppose that $\sum_{\sigma \in I} \boldsymbol{C} f_{\sigma}$ is stable under $\left[\begin{array}{rr}0 & -1 \\ N & 0\end{array}\right]_{2}$. Then the restriction of $\nu$ to $A_{0}$ is an isogeny of $A_{0}$ onto $A$, and $\nu \circ \theta_{0}(a)=\theta(a) \circ \nu$ for all $a \in K$.

Proof. Put $X=\sum_{\sigma \in I} \boldsymbol{C} f_{\sigma}$. For $h(z)=\sum_{n} b_{n} e^{2 \pi i n z}$, put $h^{*}(z)=\sum_{n} \bar{b}_{n} e^{2 \pi i n z}$. Then we see easily that $X$ is stable under the map $h \mapsto h^{*}$, and $h \mid\left[\omega_{N}\right]_{2}=\left(h \mid\left[\tau_{N}\right]_{2}\right)^{*}$. Therefore if $g=\left(h \mid\left[\tau_{N}\right]_{2}\right)^{*}, h \neq 0$, we obtain, from (2.5), $(\mu(h), \lambda(g))_{J} \neq 0$. This implies that the bilinear form $(,)_{J}$ is non-degenerate on $\mu(X) \times \lambda(X)$. It follows that $d \nu$ maps $Y\left(A_{0}\right)$ onto $Y(A)$, hence $\nu$ maps $A_{0}$ onto $A$. The relation $\nu \circ \theta_{0}(a)=\theta(a) \circ \nu$ is obvious from (ii) of Theorem 1 and (ii) of Theorem 2.

\section{$\S 3$. Periods of cusp forms.}

We first consider a general case in which $\Gamma$ is not necessarily a congruence subgroup of $S L_{2}(\boldsymbol{Z})$. Let $\Gamma$ be a discrete subgroup of $S L_{2}(\boldsymbol{R})$ such that $\mathscr{S} / \Gamma$ is of finite measure, and $\mathfrak{S}^{*}$ the union of $\mathscr{S}$ and the cusps of $\Gamma$. Further let $J$ be the jacobian variety of the compact Riemann surface $\mathfrak{S}^{*} / \Gamma$. We can then define $D_{J}, Y_{J}, S_{2}(\Gamma)$, and $\mu: S_{2}(\Gamma) \rightarrow D_{J}$ in the same fashion as in $\S 1$. Put

$$
[\gamma, g]=\int_{z}^{\gamma^{(z)}} g(z) d z
$$

for $g \in S_{2}(\Gamma)$ and $\gamma \in \Gamma$ with any $z \in \mathfrak{S}^{*}$. Note that this does not depend on the choice of $z$. Let $L_{J}$ be the submodule of $Y_{J}$ generated over $\boldsymbol{Z}$ by the linear maps 


$$
\eta \longmapsto\left[\gamma, \mu^{-1}(\eta)\right] \quad\left(\eta \in D_{J}\right)
$$

for all $\gamma \in \Gamma$. Then $J$ can be identified with $Y_{J} / L_{J}$. Fix a point $z_{0}$ of $\mathfrak{S}^{*}$, and define, for $z \in \mathfrak{H}^{*}$, an element $\varphi_{z}$ of $Y_{J}$ by

$$
\varphi_{z}(\mu(g))=\int_{z_{0}}^{z} g(z) d z \quad\left(g \in S_{2}(\Gamma)\right) .
$$

Then the map $z \mapsto \varphi_{z}$ induces a canonical map of $\mathfrak{S}^{*} / \Gamma$ into $J=Y_{J} / L_{J}$.

Let $(A, \nu)$ be a quotient of $J$ by an abelian subvariety of $J$. Take any $\boldsymbol{C}$-basis $\left\{f_{1}, \cdots, f_{m}\right\}$ of $\mu^{-1}\left(\delta \nu\left(D_{A}\right)\right)$. Let $W=Y(\operatorname{Ker}(\nu))$. Then

$$
W=\left\{v \in Y_{J} \mid\left(\delta \nu\left(D_{A}\right), v\right)_{J}=0\right\},
$$

and $A$ can be identified with $Y_{J} /\left(W+L_{J}\right)$. Now $\mu\left(f_{1}\right), \cdots, \mu\left(f_{m}\right)$ define a $\boldsymbol{C}$ linear map $F: Y_{J} \rightarrow C^{m}$ whose kernel is $W$. In this situation, we have

Proposition 3. Let $P$ be the submodule of $\boldsymbol{C}^{m}$ generated over $\boldsymbol{Z}$ by the vectors $\left(\left[\gamma, f_{1}\right], \cdots,\left[\gamma, f_{m}\right]\right)$ for all $\gamma \in \Gamma$. Then $A$ is isomorphic, via $F$, to $\boldsymbol{C}^{m} / P$.

Proof. From the above definition of $L_{J}$, we see easily that $F\left(L_{J}\right)=P$, hence our assertion.

REMARK. If we put

$$
\Phi(z)=\left(\int_{z_{0}}^{z} f_{1}(z) d z, \cdots, \int_{z_{0}}^{z} f_{m}(z) d z\right)(\bmod P),
$$

then $\Phi$ defines a morphism of $\mathfrak{H}^{*} / \Gamma$ into $C^{m} / P$. Obviously $\Phi(z)=F\left(\varphi_{z}\right)$, i. e., $\Phi$ is the map composed by a chain of maps

$$
\mathfrak{H}^{*} / \Gamma \stackrel{\varphi}{\longrightarrow} J \stackrel{\nu}{\longrightarrow} A \stackrel{F}{\longrightarrow} C^{m} / P \text {. }
$$

The above discussion naturally applies to a group $\Gamma$ of level $N$ and $(A, \nu, \theta)$ defined as in Theorem 1. In this case, we can take $\left\{f_{\sigma} \mid \sigma \in I\right\}$ as $\left\{f_{i}\right\}$. Thus we obtain the abelian variety $A$ from the periods of the cusp forms $f_{\sigma}$.

Proposition 4. Let $\lambda$ be the map of $\S 2$ defined with respect to a group $\Gamma$ of level $N$. Then $\lambda^{-1}\left(L_{J}\right)$ consists of all the elements $g$ of $S_{2}(\Gamma)$ such that $\operatorname{Re}[\gamma, g] \in Z$ for all $\gamma \in \Gamma$, where $\operatorname{Re}$ means the real part.

PROOF. First we note a relation

$$
-\overline{\left[\gamma, g \mid\left[\omega_{N}\right]_{2}\right]}=\left[\omega_{N} \gamma \omega_{N}^{-1}, g\right] \quad\left(\gamma \in \Gamma, g \in S_{2}(\Gamma)\right)
$$

which can easily be verified. Now it is well known that

$$
\begin{aligned}
-i\langle g, h\rangle & =\int_{\mathscr{S} / I} g(z) d z \wedge \operatorname{Re}(h(z) d z) \\
& =\sum_{j=1}^{p}\left\{\left[\alpha_{j}, g\right] \cdot \operatorname{Re}\left[\beta_{j}, h\right]-\left[\beta_{j}, g\right] \cdot \operatorname{Re}\left[\alpha_{j}, h\right]\right\},
\end{aligned}
$$

where $p$ is the genus of $\mathscr{H}^{*} / \Gamma$, and $\left\{\alpha_{1}, \cdots, \alpha_{p}, \beta_{1}, \cdots, \beta_{p}\right\}$ is a set of elements 
of $\Gamma$ corresponding to a standard set of generators of the homology group of $\mathfrak{S}^{*} / \Gamma$. Moreover, every $\boldsymbol{C}$-linear map of $S_{2}(\Gamma)$ into $\boldsymbol{C}$ can be given as

$$
g \longmapsto \sum_{j=1}^{p}\left(a_{j}\left[\alpha_{j}, g\right]+b_{j}\left[\beta_{j}, g\right]\right)
$$

with real numbers $a_{j}$ and $b_{j}$, which are unique for the map. Our proposition now follows immediately from these facts and (3.1).

Put $L^{\prime}=\lambda^{-1}\left(L_{J}\right)$. Let $A_{0}$ be any abelian subvariety of $J$, and let $U=$ $\lambda^{-1}\left(Y\left(A_{0}\right)\right)$. Then $A_{0}$ is isomorphic, via $\lambda$, to $U /\left(U \cap L^{\prime}\right)$. Let $\left\{g_{1}, \cdots, g_{m}\right\}$ be a $\boldsymbol{C}$-basis of $U$, and let $P_{0}$ be the submodule of $\boldsymbol{C}^{m}$ generated over $\boldsymbol{Z}$ by the vectors $\left(\left[\gamma, g_{1}\right], \cdots,\left[\gamma, g_{m}\right]\right)$ for all $\gamma \in \Gamma$. Then

Proposition 5. $A_{0}$ is dual to $\boldsymbol{C}^{m} / P_{0}$.

Proof. Define a $\boldsymbol{C}$-linear isomorphism $G: \boldsymbol{C}^{m} \rightarrow U$ by $G\left(w_{1}, \cdots, w_{m}\right)=$ $\sum_{j} w_{j} g_{j}$. By Proposition 4, $w=\left(w_{1}, \cdots, w_{m}\right) \in G^{-1}\left(U \cap L^{\prime}\right)$ if and only if $\operatorname{Re}\left(\sum_{j} w_{j}\left[\gamma, g_{j}\right]\right) \in \boldsymbol{Z}$ for all $\gamma \in \Gamma$, i. e., $\operatorname{Re}\left(\sum_{j} w_{j} v_{j}\right) \in \boldsymbol{Z}$ for all $\left(v_{1}, \cdots, v_{m}\right) \in P_{0}$. It follows that $C^{m} / G^{-1}\left(U \cap L^{\prime}\right)$ is dual to $C^{m} / P_{0}$, q. e. d.

Proposition 6. Define $(A, \nu, \theta)$ and $\left(A_{0}, \theta_{0}\right)$ as in Theorems 1 and 2 with the same eigen-function $f$. Then $A$ is dual to $A_{0}$.

PROOF. In this case $\lambda^{-1}\left(Y\left(A_{0}\right)\right)=\sum_{\sigma \in I} C f_{\sigma}=\mu^{-1}\left(\delta \nu\left(D_{A}\right)\right)$, so that we can take $P=P_{0}$. Then our assertion is immediate from Propositions 3 and 5.

\section{$\S 4$. A twisting operator $R$ and its geometric meaning.}

Let $\chi$ be a primitive character modulo a positive integer $r$, and let $\alpha_{u}=$ $\left[\begin{array}{cc}1 & u / r \\ 0 & 1\end{array}\right]$ for $u \in Z$. Define an operator $R$ by

$$
f\left|R=\sum_{u=1}^{r} \bar{\chi}(u) f\right|\left[\alpha_{u}\right]_{2} \quad\left(f \in S_{2}\left(\Gamma_{1}(N)\right)\right) .
$$

By [11, Prop. 3.64], $R$ maps $S_{2}\left(\Gamma_{1}(N)\right)$ into $S_{2}\left(\Gamma_{1}\left(r^{2} N\right)\right)$. If $f(z)=\sum_{n} a_{n} e^{2 \pi i n z}$, then $f \mid R=g(\bar{\chi}) \sum_{n} \chi(n) a_{n} e^{2 \pi i n z}$, where $g(\bar{\chi})=\sum_{u=1}^{r} \bar{\chi}(u) e^{2 \pi i u / r}$. The purpose of this section is to study the geometric meaning of $R$ in connection with the quotients $(A, \nu)$ of $J$.

Let $s$ be a positive divisor of $N$, and $\mathfrak{h}$ a subgroup of $(\boldsymbol{Z} / s \boldsymbol{Z})^{\times}$. Define a group $\Gamma$ of level $N$ by

$$
\Gamma=\left\{\left[\begin{array}{ll}
a & b \\
c & d
\end{array}\right] \in \Gamma_{0}(N) \mid d(\bmod s) \in \mathfrak{h}\right\} .
$$

Let $M$ be a common multiple of $N, r^{2}$, and $r s$. Let $\Gamma^{\prime}$ be a group of level $M$ such that

$$
\Gamma^{\prime} \subset\left\{\left[\begin{array}{ll}
a & b \\
c & d
\end{array}\right] \in \Gamma_{0}(M) \mid d(\bmod s) \in \mathfrak{h}, a \equiv d(\bmod r)\right\} .
$$


Then $\alpha_{u} \Gamma^{\prime} \alpha_{u}^{-1} \subset \Gamma$, and $R$ maps $S_{2}(\Gamma)$ into $S_{2}\left(\Gamma^{\prime}\right)$.

Define $J, J^{\prime}, \mu, \mu^{\prime}, \lambda, \lambda^{\prime}$ as before with respect to the present $\Gamma$ and $\Gamma^{\prime}$. Since $f\left|\left[\alpha_{u}\right]_{2}=f\right|\left[\Gamma \alpha_{u} \Gamma^{\prime}\right]_{2}$ for $f \in S_{2}(\Gamma)$, we have

$$
\begin{aligned}
& \mu^{\prime}(f \mid R)=\sum_{u=1}^{r} \bar{\chi}(u) \cdot \delta\left\{\Gamma \alpha_{u} \Gamma^{\prime}\right\}(\mu(f)) \\
& \lambda^{\prime}(f \mid R)=\sum_{u=1}^{r} \bar{\chi}(u) \cdot d\left\{\Gamma^{\prime} \beta_{u} \Gamma\right\}(\lambda(f))
\end{aligned} \quad\left(f \in S_{2}(\Gamma)\right),
$$

where $\beta_{u}=\omega_{M}^{-1} \alpha_{u}^{e} \omega_{N}$.

PROPOSITION 7. Let $f(z)=\sum_{n} a_{n} e^{2 \pi i n z}$, with $a_{1}=1$, be a common eigenfunction of $T_{n}$ in $S_{2}(\Gamma)$ for all $n$. Define $(A, \nu, \theta)$ and $\left(A^{\prime}, \nu^{\prime}, \theta^{\prime}\right)$ for $f$ and $h(z)=\sum_{n} \chi(n) a_{n} e^{2 \pi i n z}$, respectively, as in Theorem 1 . Then $A^{\prime}$ is a homomorphic image of the product of $r$ copies of $A$.

Proof. Let $K^{\prime \prime}$ be the subfield of $\boldsymbol{C}$ generated by the Fourier coefficients $a_{n}$ and the values $\chi(n)$ for all $n$, and let $I^{\prime \prime}$ be the set of all isomorphisms of $K^{\prime \prime}$ into $C$. For $\sigma \in I^{\prime \prime}$, put $f_{\sigma}(z)=\sum_{n} a_{n}^{\sigma} e^{2 \pi i n z}, h_{\sigma}(z)=\sum_{n} \chi(n)^{\sigma} a_{n}^{\sigma} e^{2 \pi i n z}$. Then $\mu\left(\sum_{\sigma \in I^{\prime \prime}} C f_{\sigma}\right)=\delta \nu\left(D_{A}\right)$, and

$$
\delta \nu^{\prime}\left(D_{A^{\prime}}\right)=\mu^{\prime}\left(\sum_{\sigma \in I^{\prime \prime}} C h_{\sigma}\right) \subset \sum_{u=1}^{r} \delta\left\{\Gamma \alpha_{u} \Gamma^{\prime}\right\} \delta \nu\left(D_{A}\right) .
$$

Define a homomorphism $\xi: J^{\prime} \rightarrow A \times \cdots \times A$ ( $r$ copies $)$ by $\xi(x)=\left(\nu\left(\left\{\Gamma \alpha_{1} \Gamma^{\prime}\right\} x\right)\right.$, $\left.\cdots, \nu\left(\left\{\Gamma \alpha_{r} \Gamma^{\prime}\right\} x\right)\right)$ for $x \in J^{\prime}$. Then (4.4) shows that the identity component of $\operatorname{Ker}(\xi)$ is contained in $\operatorname{Ker}\left(\nu^{\prime}\right)$. Therefore we can find a homomorphism $\beta: A \times \cdots \times A \rightarrow A^{\prime}$ such that $\beta \circ \xi=m \nu^{\prime}$ with a positive integer $m$. This proves our proposition.

Let us now assume that $\chi$ is a non-trivial real character. Put

$$
\eta_{0}=\sum_{u=1}^{r} \chi(u)\left\{\Gamma \alpha_{u} \Gamma^{\prime}\right\}
$$

Then $\eta_{0} \in \operatorname{Hom}\left(J^{\prime}, J\right)$, and

$$
\mu^{\prime}(g \mid R)=\delta \eta_{0}(\mu(g)) \quad\left(g \in S_{2}(\Gamma)\right) .
$$

Proposition 8. Let $A$ and $A^{\prime}$ be as in Proposition 7. Then there exists a homomorphism $\eta$ of $A^{\prime}$ into $A$ such that $\eta \circ \nu^{\prime}=\nu \circ \eta_{0}$. The homomorphisms $\eta_{0}$ and $\eta$ are defined over the quadratic extension $\mathfrak{f}$ of $\boldsymbol{Q}$ corresponding to $\chi$, and $\eta_{0}^{\varepsilon}=-\eta_{0}, \eta^{\varepsilon}=-\eta$ if $\varepsilon$ is the generator of $\mathrm{Gal}(\mathfrak{f} / \mathbf{Q})$.

Proof. We need the precise definition of "the standard models" for $\mathfrak{S}^{*} / \Gamma$ and $\mathfrak{S}^{*} / \Gamma^{\prime}$. Using the notation of $[11, \S 7.3]$, let us define subgroups $U_{N}$ and $S$ of $G_{A}$ by

$$
\begin{aligned}
& U_{N}=\left\{x \in \prod_{p} G L_{2}\left(\boldsymbol{Z}_{p}\right) \times G_{\infty+} \mid x_{p} \equiv\left[\begin{array}{ll}
* & * \\
0 & 1
\end{array}\right](\bmod N)\right\}, \\
& S=\boldsymbol{Q}^{\times} U_{N} \Gamma .
\end{aligned}
$$


Similarly put $S^{\prime}=\boldsymbol{Q}^{\times} U_{M} \Gamma^{\prime}$. Then $G_{\boldsymbol{Q}} \cap S=\boldsymbol{Q}^{\times} \Gamma, G_{\boldsymbol{Q}} \cap S^{\prime}=\boldsymbol{Q}^{\times} \Gamma^{\prime}$; we take $V_{S}, V_{S^{\prime}}$ of $\left[11\right.$, p. 155] as models of $\mathfrak{S}^{*} / \Gamma, \mathfrak{S}^{*} / \Gamma^{\prime}$; and $X\left(\Gamma \alpha_{u} \Gamma^{\prime}\right)=X_{S S^{\prime}}\left(\alpha_{u}\right)$. Now one can easily verify that

$$
\boldsymbol{Q}^{\times} \cdot \operatorname{det}\left(S^{\prime} \cap \alpha_{u}^{-1} S \alpha_{u}\right) \supset \boldsymbol{Q}^{\times} \cdot \boldsymbol{R}_{+}^{\times} \cdot\left\{\left(v_{p}\right) \in \prod_{p} \boldsymbol{Z}_{p}^{\times} \mid v_{p} \equiv 1(\bmod r)\right\} .
$$

By [11, Prop. 7.2], this shows that $X_{S S^{\prime}}\left(\alpha_{u}\right)$ is rational over $\boldsymbol{Q}\left(\zeta_{r}\right)$, where $\zeta_{r}=e^{2 \pi i / r}$. For an integer $q$ prime to $M$, let $\sigma_{q}$ be an element of $S L_{2}(\boldsymbol{Z})$ such that $q \cdot \sigma_{q} \equiv\left[\begin{array}{ll}1 & 0 \\ 0 & q^{2}\end{array}\right](\bmod M)$. If $g \in S_{2}(\Gamma)$ and $g \mid\left[\sigma_{q}\right]_{2}=\varphi(q) g$ with a character $\varphi$ modulo $s$, then, by $\left[11\right.$, Prop. 3.64], one has $(g \mid R) \mid\left[\sigma_{q}\right]_{2}=$ $\varphi(q) g\left|R=\left(g \mid\left[\sigma_{q}\right]_{2}\right)\right| R$. This shows that

$$
\eta_{0} \circ\left\{\Gamma^{\prime} \sigma_{q} \Gamma^{\prime}\right\}=\left\{\Gamma \sigma_{q} \Gamma\right\} \circ \eta_{0} .
$$

On the other hand, let $y=\left(y_{p}\right)$ be an element of $G_{0}$ such that $y_{p}=\left[\begin{array}{ll}1 & 0 \\ 0 & q\end{array}\right]$ or 1 according as $p$ divides $M$ or not. Then $\sigma_{q}^{-1} y \in U_{M}$ so that $J_{S S}\left(\sigma_{q}\right)=J_{S S}(y)$, $J_{S^{\prime} S^{\prime}}\left(\sigma_{q}\right)=J_{S^{\prime} S^{\prime}}(y)$. Since $\alpha_{u}\left[\begin{array}{ll}1 & 0 \\ 0 & q\end{array}\right]=\left[\begin{array}{ll}1 & 0 \\ 0 & q\end{array}\right] \alpha_{q u}$, we obtain, by [11, Prop. 7.2],

$$
\sum_{u=1}^{r} \chi(q u) X_{S S^{\prime}}\left(\alpha_{u}\right)^{\sigma(y)} \circ J_{S^{\prime} S^{\prime}}(y)=J_{S S}(y) \circ\left(\sum_{u=1}^{r} \chi(q u) X_{S S^{\prime}}\left(\alpha_{q u}\right)\right),
$$

where $\sigma(y)$ is the element of $\operatorname{Gal}\left(\boldsymbol{Q}_{a b} / \boldsymbol{Q}\right)$ defined by $[\mathbf{1 1},(6.4 .1)]$, hence $\chi(q) \eta_{0}^{\sigma(y)} \circ\left\{\Gamma^{\prime} \sigma_{q} \Gamma^{\prime}\right\}=\left\{\Gamma \sigma_{q} \Gamma\right\} \circ \eta_{0}$. Combining this with (4.7), we obtain

$$
\eta_{0}^{\sigma(y)}=\chi(q) \eta_{0} \text {. }
$$

Observe that $\sigma(y)$ sends $\zeta_{M}=e^{2 \pi i / M}$ onto $\zeta_{M}^{q}$. Therefore $\eta_{0}$ is rational over the quadratic field $\mathfrak{f}$ corresponding to $\chi$, and $\eta_{0}^{\varepsilon}=-\eta_{0}$ for the generator $\varepsilon$ of $\operatorname{Gal}(\mathfrak{t} / \boldsymbol{Q})$. Now $R$ maps $\sum_{\sigma \in I^{\prime \prime}} \boldsymbol{C} f_{\sigma}$ into $\sum_{\sigma \in I^{\prime \prime}} \boldsymbol{C} h_{\sigma}$, so that $\delta \eta_{0}$ maps $\delta \nu\left(D_{A}\right)$ into $\delta \nu^{\prime}\left(D_{A^{\prime}}\right)$. Therefore we can define a homomorphism $\eta$ of $A^{\prime}$ into $A$, rational over $\mathfrak{l}^{\prime}$, so that $\eta \circ \nu^{\prime}=\nu \circ \eta_{0}$. Then the relation $\eta^{\varepsilon}=-\eta$ is obvious.

Let us now assume the following set of conditions:

$$
\begin{aligned}
& \text { (i) } N \text { is a common multiple of } r^{2} \text { and } r s \text {; (ii) } a \equiv d(\bmod r) \text { for } \\
& \text { every }\left[\begin{array}{ll}
a & b \\
c & d
\end{array}\right] \in \Gamma \text {. }
\end{aligned}
$$

Then we can take $M=N, \Gamma^{\prime}=\Gamma, J^{\prime}=J$, so that $\eta_{0}$ is an endomorphism of $J$, and $\eta_{0}^{3}=\chi(-1) r \eta_{0}$.

Fix an eigen-function $f(z)$ as in Proposition 6, and define $K$ and $(A, \nu, \theta)$ as before. Suppose that the following condition is satisfied:

(4.9) There is an automorphism $\rho$ of $K$, other than the identity map, such that $\chi(n) a_{n}=a_{n}^{\rho}$ for all $n$. (This implies especially that $a_{n}=0$ if $(n, r) \neq 1$.) 
Then $\rho^{2}=1$, and $\chi(n) a_{n}^{\sigma}=a_{n}^{\rho \sigma}$ for any $\sigma \in I$, so that

$$
f_{\sigma} \mid R=g(\chi) f_{\rho \sigma} .
$$

Proposition 9. Under the assumptions (4.8) and (4.9), $\eta$ is an endomorphism of $A$ satisfying

(i) $\eta^{2}=\chi(-1) r \cdot i d_{A}$,

(ii) $\eta \circ \theta(a)=\theta\left(a^{\rho}\right) \circ \eta$ for every $a \in K$.

Proof. Observe that the diagram

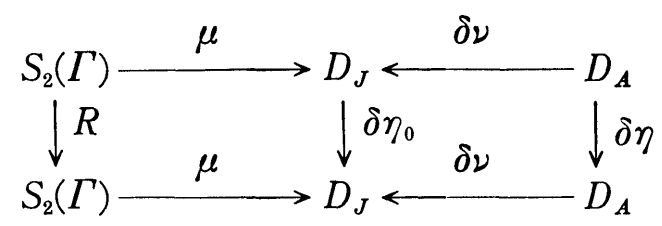

is commutative, and another diagram with $T_{n}, \delta \xi_{n}, \delta \theta\left(a_{n}\right)$ in place of $R, \delta \eta_{0}$, $\delta \eta$ is commutative, too. Let $w_{\sigma}$ be the element of $D_{A}$ such that $\delta \nu\left(w_{\sigma}\right)=\mu\left(f_{\sigma}\right)$. Then $\left\{w_{\sigma} \mid \sigma \in I\right\}$ is a $\boldsymbol{C}$-basis of $D_{A}$, and the commutativity of the diagram implies $\delta \eta\left(w_{\sigma}\right)=\mathrm{g}(\chi) w_{\rho \sigma}$, and $\delta \theta(a)\left(w_{\sigma}\right)=a^{\sigma} w_{\sigma}$ for $a \in K$. Therefore $\delta \eta^{2}\left(w_{\sigma}\right)=$ $\mathfrak{g}(\chi)^{2} w_{\sigma}$, and $(\delta \eta \circ \delta \theta(a))\left(w_{\sigma}\right)=\mathfrak{g}(\chi) a^{\sigma} w_{\rho \sigma}=\left(\delta \theta\left(a^{\rho}\right) \circ \delta \eta\right)\left(w_{\sigma}\right)$, hence our proposition.

Thus, if an eigen-function $f(z)$ satisfying (4.9) exists, then the corresponding $(A, \theta)$ and $\eta$ form a system similar to that of $[13, \S 9]$. The field $K$ in the present case is not necessarily a $C M$-field as assumed there, but one can still develop, by the same ideas as in $[13, \S 9]$, a theory of construction of class fields over the quadratic field $\mathrm{f}$. As mentioned in the Introduction, $\mathrm{K}$. Doi and M. Yamauchi have found a few examples of $f(z)$, satisfying (4.9), with $N=p^{3}$ and $r=p$ for a prime $p$.

\section{§ 5. A proof of Dirichlet's class number formula.}

Let $\boldsymbol{Q}(\sqrt{-q})$ be an imaginary quadratic field with discriminant $-q$, and $h(-q)$ the class number of $\boldsymbol{Q}(\sqrt{-q})$. Then the Dirichlet formula is, if $q>4$,

$$
h(-q)=-\sum_{n=1}^{q}\left(\frac{-q}{n}\right) n / q \text {. }
$$

The purpose of this section is to prove this as an application of the results of previous sections, without using the residue of the zeta function of $\boldsymbol{Q}(\sqrt{-q})$. The proof is inspired by a result of Hecke's in [5]; we shall explain its connection with our proof afterwards.

For simplicity, we assume $q>4$, although the cases $q=3,4$ can be included, without much trouble, in the following treatment. Put $\chi(n)=\left(\frac{-q}{n}\right)$, and 


$$
\Gamma=\left\{\left[\begin{array}{ll}
a & b \\
c & d
\end{array}\right] \in \Gamma_{0}\left(q^{2}\right) \mid a \equiv d \equiv 1(\bmod q)\right\} .
$$

Define the operator $R$ of $\S 4$ for this $\chi$ with $N=q^{2}, r=q$. Since the present $\Gamma$ satisfies (4.8), $R$ maps $S_{2}(\Gamma)$ into itself, and

$$
\left(\sum_{n=1}^{\infty} a_{n} e^{2 \pi i n z}\right) \mid R=\sqrt{-q} \sum_{n=1}^{\infty} \chi(n) a_{n} e^{2 \pi i n z} .
$$

Here we recall a well-known formula of Gauss

$$
\sum_{n=1}^{q} \chi(n) e^{2 \pi i n k / q}=\sqrt{-q} \cdot \chi(k) .
$$

We understand that $\sqrt{-q}$ has positive imaginary part.

Proposition 10. One has $\operatorname{tr}(R)=\sqrt{-q} \cdot h(-q) \cdot \varphi(q) / 2$, where $\varphi$ is Euler's function.

PROOF. By virtue of the results of Miyake [8] and Casselman [2] (which generalize Atkin-Lehner [1]), we can find a basis of $S_{2}(\Gamma)$ formed by the elements

$$
g_{1}, \cdots, g_{s}, g_{1}(m z), \cdots, g_{s}(m z),
$$

where $g_{k}$ belongs to "the essential part" of $S_{2}\left(\Gamma_{1}\left(N_{k}\right)\right)$ for a divisor $N_{k}$ of $q^{2}$, and $m$ runs over all non-trivial positive divisors of $q^{2} / N_{k}$. (In other words, $g_{k}$ is a "new form".) We take $g_{k}$ to be a common eigen-function of all Hecke operators of level $N_{k}$, with the first Fourier coefficient 1 . If $g_{k}(z)=\sum_{n=1}^{\infty} a_{n} e^{2 \pi i n z}$, we see that the $g_{k}(m z)$, for a fixed $k$ and for all positive divisors of $q^{2} / N_{k}$, span, over $C$, the vector space

$$
\left\{f \in S_{2}(\Gamma)|f| T_{n}=a_{n} f \text { for all } n \text { prime to } q\right\} \text {. }
$$

Observe that $R$ maps $g_{k}(m z)$ onto 0 if $m>1$, and $g_{k}$ onto a common eigenfunction of all Hecke operators of level $q^{2}$, since $\sum_{n} \chi(n) a_{n} n^{-s}$ has an Euler product. Such an eigen-function must be of the form $\sum_{m} c_{m} g_{j}(m z)$ for some $j$, where $m$ divides $q^{2} / N_{j}$. The comparison of the first Fourier coefficients yields $c_{1}=\sqrt{-q}$. Therefore $\operatorname{tr}(R) / \sqrt{-q}$ is the number of indices $k$ such that

$$
g_{k} \mid R=\sqrt{-q} \cdot g_{k}+\sum_{m} c_{m} g_{k}(m z) \quad\left(m>1, m \mid N_{k}^{-1} q^{2}\right) .
$$

Put $g_{k}^{*}(z)=\sum_{(n, q)=1} a_{n} e^{2 \pi i n z}$. Since $\left(g_{k} \mid R\right) \mid T_{n}=a_{n} \cdot\left(g_{k} \mid R\right)$ for $(n, q)=1$, and the $n$-th Fourier coefficient of $g_{k} \mid R$ is 0 for $(n, q) \neq 1$, we have $g_{k}\left|R=g_{k}^{*}\right| R=$ $\sqrt{-q} \cdot g_{k}^{*}$. Define $(A, \nu), I$, and $f_{\sigma}$ as in Theorem 1 with $g_{k}^{*}$ as $f$. By Proposition 8 , there is an endomorphism $\eta$ of $A$ such that

$$
\delta \eta\left(\delta \nu^{-1}\left(\mu\left(f_{\sigma}\right)\right)\right)=\delta \nu^{-1}\left(\mu\left(f_{\sigma} \mid R\right)\right)
$$


for all $\sigma \in I$. Since $f_{\sigma} \mid R=\sqrt{-q} \cdot f_{\sigma}$, we see that $\delta \eta$ is $\sqrt{-q}$ times the identity map on $D_{A}$. Therefore, by [12, Lemma 1], $A$ is isogenous to the product of copies of an elliptic curve, say $E$, with complex multiplication, such that End $(E) \otimes \boldsymbol{Q}$ is isomorphic to $\boldsymbol{Q}(\sqrt{-q})$. Now define an abelian variety $B$ for the function $g_{k}$ (at level $N_{k}$ ) by Theorem 1. By Proposition 8 , $B$ is isogenous to $A$, hence $B$ is also isogenous to the product of copies of $E$. Then, by [13, Prop. 1.6], $g_{k}$ must be the Mellin (inverse) transform of an $L$-function with a primitive Grössen-character $\lambda$ of $\boldsymbol{Q}(\sqrt{-q})$. This means that $g_{k}(z)=\sum_{a} \lambda(\mathfrak{a}) e^{2 \pi i_{N(a) z}}$, where $a$ runs over all integral ideals in $\boldsymbol{Q}(\sqrt{-q})$ prime to the conductor, say $\mathrm{c}$, of $\lambda$, and $\lambda((\alpha))=\alpha$ for $\alpha \equiv 1 \bmod$ c. Moreover the functional equation of the $L$-function implies that $g_{k}$ belongs to the essential part of $S_{2}\left(\Gamma_{1}(q \cdot N(\mathfrak{c}))\right)$, i. e., it is a "new form". Therefore $N(\mathfrak{c})$ divides $q$, hence $c$ divides the ideal $(\sqrt{-q})$. It can easily be seen that there are exactly $h(-q) \cdot \varphi(q) / 2$ primitive Grössen-characters $\phi$ whose conductor $c$ divides $\sqrt{-q}$ and such that $\phi((\alpha))=\alpha$ for $\alpha \equiv 1 \bmod$. For any such $\phi$, put $f_{\phi}(z)=$ $\sum_{a} \phi(\mathfrak{a}) e^{2 \pi i_{N}(\mathfrak{a}) z}$, where $\mathfrak{a}$ runs over all integral ideals of $\boldsymbol{Q}(\sqrt{-q})$ prime to the conductor of $\phi$. Then the $f_{\psi}$, for all such $\phi$, are linearly independent, and belong to $S_{2}(\Gamma)$, by [12, Lemma 3]. Further each $f_{\phi}$ must coincide with one of the $g_{k}$, and obviously $f_{\phi} \mid R=\sqrt{-q} \cdot f_{\psi}^{*}$. Thus we have shown that $\operatorname{tr}(R) / \sqrt{-q}$ is the number of the characters $\phi$, which completes the proof. and

To compute $\operatorname{tr}(R)$ in a different manner, put $\alpha=\left[\begin{array}{ll}1 & 0 \\ 0 & q\end{array}\right], \varepsilon=\left[\begin{array}{ll}1 & 1 \\ 0 & 1\end{array}\right]$,

$$
\Gamma(q)=\left\{\left[\begin{array}{ll}
a & b \\
c & d
\end{array}\right] \in S L_{2}(\boldsymbol{Z}) \mid\left[\begin{array}{ll}
a & b \\
c & d
\end{array}\right] \equiv\left[\begin{array}{ll}
1 & 0 \\
0 & 1
\end{array}\right](\bmod q)\right\} .
$$

Then $\Gamma=\alpha \Gamma(q) \alpha^{-1}, \alpha \varepsilon \alpha^{-1}=\left[\begin{array}{cc}1 & 1 / q \\ 0 & 1\end{array}\right]$. Therefore $\operatorname{tr}(R)$ is equal to the trace of the operator

$$
f \longmapsto \sum_{u=1}^{q} \chi(u) f \mid\left[\varepsilon^{u}\right]_{2}
$$

on the space $S_{2}(\Gamma(q))$ of cusp forms of weight 2 with respect to $\Gamma(q)$. Let $\varepsilon_{0}$ be the automorphism of $\mathfrak{S}^{*} / \Gamma(q)$ obtained from $\varepsilon$. The fixed points of $\varepsilon_{0}$ occur only at cusps. Indeed, suppose $\varepsilon(z)=\gamma(z)$ for some $z \in \mathscr{S}$ and some $\gamma=\left[\begin{array}{ll}a & b \\ c & d\end{array}\right] \in \Gamma(q)$. Then $\varepsilon^{-1} \gamma=\left[\begin{array}{cc}a-c & b-d \\ c & d\end{array}\right]$ is elliptic, hence $\left|\operatorname{tr}\left(\varepsilon^{-1} \gamma\right)\right|<2$. But $a-c+d \equiv 2(\bmod q)$, which is a contradiction, since $q>4$.

Now let $r / s$, with integers $r$ and $s$ such that $(r, s)=1$, be a cusp representing a fixed point of $\varepsilon_{0}$. By [11, Lemma 1.42], this is so if and only if $\left[\begin{array}{l}r \\ s\end{array}\right] \equiv \pm\left[\begin{array}{c}r+s \\ s\end{array}\right](\bmod q)$. Therefore $\varepsilon_{0}$ has exactly $\varphi(q) / 2$ fixed points represented by $r / q$ with $(r, q)=1,0<r<q / 2$. For such an $r$, take an element $\beta$ 
of $S L_{2}(\boldsymbol{Z})$ of the form $\beta=\left[\begin{array}{rr}u & v \\ -q & r\end{array}\right]$. Then $\beta$ maps $r / q$ to $\infty$, and

$$
\beta^{-1}\left[\begin{array}{rr}
1 & m \\
0 & 1
\end{array}\right] \beta=\left[\begin{array}{rr}
1-m q r & m r^{2} \\
-m q^{2} & 1+m q r
\end{array}\right] \text {. }
$$

Choose an integer $m$ so that $m r^{2} \equiv 1(\bmod q)$. Then $\beta^{-1}\left[\begin{array}{cc}1 & m \\ 0 & 1\end{array}\right] \beta \varepsilon^{-1} \in \Gamma(q)$, so that $\beta^{-1}\left[\begin{array}{cc}1 & m \\ 0 & 1\end{array}\right] \beta$ represents the automorphism $\varepsilon_{0}$, and moreover it has $r / q$ as a fixed point. Therefore, if $t$ is the local parameter on $\mathscr{S}^{*} / \Gamma(q)$ around $r / q$ defined by

$$
t(z)=\exp [2 \pi i \cdot \beta(z) / q],
$$

one has $t \circ \varepsilon_{0}=\zeta^{m} t$ with $\zeta=e^{2 \pi i / q}$. Let $\operatorname{tr}\left(\varepsilon_{0}\right)$ denote the trace of $[\varepsilon]_{2}$ on $S_{2}(\Gamma(q))$. Applying the Selberg-Eichler trace formula to $\varepsilon_{0}$, we obtain

$$
\operatorname{tr}\left(\varepsilon_{0}^{u}\right)-1=2^{-1} \sum_{(r, q)=1} \zeta^{r^{2} u} /\left(1-\zeta^{r^{2} u}\right),
$$

for $(u, q)=1$. Therefore

$$
\operatorname{tr}(R)=\sum_{u=1}^{q-1} \chi(u) \operatorname{tr}\left(\varepsilon_{0}^{u}\right)=2^{-1} \varphi(q) \sum_{m=1}^{q-1} \chi(m) \zeta^{m} /\left(1-\zeta^{m}\right) .
$$

To compute the last sum, take an indeterminate $w$, and observe that

$$
\begin{aligned}
\sum_{m=1}^{q-1} \chi(m) \zeta^{m} /\left(1-\zeta^{m} w\right) & =\sum_{k=1}^{\infty} w^{k-1} \sum_{m=1}^{q-1} \chi(m) \zeta^{m k} \\
& =\sqrt{-q} \sum_{k=1}^{\infty} \chi(k) w^{k-1} \\
& =\sqrt{-q}\left(\sum_{k=1}^{q-1} \chi(k) w^{k-1}\right) /\left(1-w^{q}\right)
\end{aligned}
$$

hence

$$
\begin{aligned}
\operatorname{tr}^{-}(R) & =\left(\sqrt{-q_{i}} \cdot \varphi(q) / 2\right) \cdot \lim _{w \rightarrow 1}\left(\sum_{k=1}^{q-1} \chi(k) w^{k}\right) /\left(1-w^{q}\right) \\
& =-(\sqrt{-q} \cdot \varphi(q) / 2 q) \sum_{k=1}^{q-1} \chi(k) k,
\end{aligned}
$$

by differentiating the numerator and the denominator. Combining this with Proposition 10, we obtain Dirichlet's formula (5.1).

Especially, suppose $q$ is a prime, and denote by $U$ the right hand side of (5.3), Then $U+\bar{U}=-\varphi(q) / 2$, and

so that

$$
U-\bar{U}=\sum_{m=1}^{q-1} \chi(u m) \zeta^{m} /\left(1-\zeta^{m}\right)=-\sqrt{-q} \cdot \chi(u) \sum_{k=1}^{q-1} \chi(k) k / q,
$$

$$
\operatorname{tr}\left(\varepsilon_{0}^{u}\right)-1=-\varphi(q) / 4-\sqrt{-q} \cdot \chi(u) \sum_{k=1}^{q-1} \chi(k) k / 2 q .
$$


Let us now clarify the relation between our proof and Hecke's results in [4], [5]. Put $\mathfrak{M}_{q}=S L_{2}(\boldsymbol{Z} / q \boldsymbol{Z}) /\{ \pm 1\}$, and suppose that $q$ is a prime such that $q \equiv 3(\bmod 4)$ and $q>3$. Then $\mathfrak{M}_{q}$ has an irreducible representation $\mathbb{G}$ :of degree $(q-1) / 2$ such that

$$
\operatorname{tr}(\mathbb{B}(\varepsilon))=(-1+\sqrt{-q}) / 2 .
$$

Let $\mathscr{D}$ be the representation of $\mathfrak{M}_{q}$ on $S_{2}(\Gamma(q))$ via the map $\gamma \mapsto[\gamma]_{2}$ for $\gamma \in S L_{2}(Z)$. Let $y_{1}$ and $y_{2}$ be the multiplicities of $\mathbb{S}$ and its complex conjugate $\mathbb{\mathbb { S }}$ in $\mathfrak{D}$, respectively. In [5], Hecke proved

$$
y_{1}-y_{2}=h(-q)
$$

by first showing

$$
y_{1}-y_{2}=-\sum_{n=1}^{q-1} \chi(n) n / q
$$

and employing Dirichlet's formula (5.1), Actually Proposition 10, combined with a simple observation, leads to (5.5) with neither Eichler-Selberg's trace formula nor Dirichlet's formula, but with the knowledge of all irreducible representations of $\mathfrak{M}_{q}$. To see this, put $\omega=\left[\begin{array}{cc}1 & 1 / q \\ 0 & 1\end{array}\right]$, and take a basis of $S_{2}(\Gamma)$ formed by eigen-functions of $[\omega]_{2}$, and let $f$ be a member of the basis. Then we have $f \mid[\omega]_{2}=\zeta^{m} f$ with $\zeta=e^{2 \pi i / q}$ and $0 \leqq m<q$, hence

$$
f \mid R=\sum_{k=1}^{q-1} \chi(k) \zeta^{m k} f=\chi(m) \sqrt{-q} \cdot f .
$$

Now, for any irreducible representation $\mathbb{S} *$ of $\mathfrak{M}_{q}$ other than $\mathbb{B}$ and $\overline{\mathbb{S}}$, $\operatorname{tr}(\mathbb{B} *(\varepsilon))$ is a rational integer (see, e.g., Hecke $[4$, p. 529 (Werke)]). Therefore we have

$$
\begin{aligned}
\operatorname{tr}\left([\omega]_{2}\right) & =\operatorname{tr}(\mathscr{D}(\varepsilon)) \\
& =y_{1} \cdot \sum_{\chi(m)=1} \zeta^{m}+y_{2} \cdot \sum_{\chi(m)=-1} \zeta^{m}+a \zeta^{0}+b \sum_{m=1}^{q-1} \zeta^{m}
\end{aligned}
$$

with non-negative integers $a$ and $b$. Here each term $\zeta^{m}(m \geqq 0)$ corresponds to a member of our basis, and by (5.7), contributes $\chi(m) \sqrt{-q}$ to $\operatorname{tr}(R)$, hence

$$
\operatorname{tr}(R)=\left(y_{1}-y_{2}\right) \sqrt{-q}(q-1) / 2 .
$$

Comparing this with Proposition 10, we obtain (5.5).

We also notice that (5.6) can be derived from (5.4) and (5.8) by comparing the imaginary parts, $\operatorname{since} \operatorname{tr}\left(\varepsilon_{0}\right)=\operatorname{tr}(\mathfrak{D}(\varepsilon))$. Actually Hecke obtained (5.6) by computing the dimension of the vector space

$$
\left\{f \in S_{2}(\Gamma(q))|f|[\varepsilon]_{2}=\zeta^{v} f\right\} \quad(0 \leqq v<q)
$$


by means of the Riemann-Roch theorem. For the reader's convenience, let us now compute $\operatorname{tr}(R)$ by Hecke's technique, using the Riemann-Roch theorem instead of the Selberg-Eichler trace formula.

Let $\lambda_{v}$ denote the dimension of $(5.9)$, Then

$$
\operatorname{tr}(R)=\sum_{k=1}^{q-1} \chi(k) \operatorname{tr}\left(\varepsilon_{0}^{k}\right)=\sum_{k=1}^{q-1} \sum_{v=0}^{q-1} \chi(k) \zeta^{k v} \lambda_{v}=\sqrt{-q} \cdot \sum_{v=1}^{q-1} \chi(v) \lambda_{v} .
$$

Put $W=\mathfrak{S}^{*} / \Gamma(q)$ and $W^{\prime}=\mathfrak{S}^{*} / \Gamma_{1}(q)$. For every positive divisor $n$ of $q$, let $P_{i}^{n}$, for $i=1, \cdots, c_{n}$, denote all the points of $W^{\prime}$ with ramification index $q / n$, and $Q_{i 1}^{n}, \cdots, Q_{i n}^{n}$ the points of $W$ lying above $P_{i}^{n}$. Put $q_{n}=q / n$. Let $g$ and $\mathfrak{g}^{\prime}$ be the genera of $W$ and $W^{\prime}$, respectively. Then

$$
2 \mathfrak{g}-2=\left(2 g^{\prime}-2\right) q+\sum_{n \mid q}\left(q_{n}-1\right) n c_{n} .
$$

We can find a $\Gamma(q)$-invariant automorphic form $f_{0}$ of weight 2 such that $f_{0} \mid[\varepsilon]_{2}=\zeta^{v} f_{0}$ with any integer $v$. Let $\operatorname{div}(X) \operatorname{resp} \operatorname{div}^{\prime}(X)$ denote the divisor of a function or a differential form $X$ on $W$ resp. $W^{\prime}$. Then

$$
\operatorname{div}\left(f_{0}(z) d z\right)=D+\sum_{n \mid q} \sum_{i=1}^{c_{n}} k_{n i}\left(Q_{i 1}^{n}+\cdots+Q_{i n}^{n}\right)
$$

with integers $k_{n i}$ and a divisor $D$ on $W$ not involving $Q_{i j}^{n}$. Let $t_{n i}$ be a local parameter on $W$ at $Q_{i 1}^{n}$. Then

$$
t_{n i} \circ \varepsilon_{0}^{n}=\zeta^{n h} t_{n i}+(\text { higher terms) }
$$

with an integer $h$ prime to $q_{n}$. Then we see that $n v \equiv n h\left(k_{n i}+1\right)(\bmod q)$. Take an integer $s_{n i}$ so that $s_{n i} h \equiv 1\left(\bmod q_{n}\right)$. Note that $s_{n i}$ is independent of $v$. Then $k_{n i} \equiv s_{n i} v-1\left(\bmod q_{n}\right)$. Especially, if $n=1$, the above study of the fixed points of $\varepsilon_{0}$ shows that $c_{1}=\varphi(q) / 2$, and $P_{i}^{1}$ is represented by $r / q$ under the conditions $(r, q)=1,0<r<q / 2$. For this point, we can take $s_{1 i}=r^{2}$, as seen above. Now the map $f \mapsto F=f_{0}^{-1} f$ sends the vector space (5.9) isomorphically onto the vector space of all $\Gamma_{1}(q)$-invariant modular functions $F$ such that

$$
\operatorname{div}(F) \geqq-\operatorname{div}\left(f_{0} d z\right) .
$$

We see easily that this is so if and only if

$$
\operatorname{div}^{\prime}(F) \geqq-q^{-1} D^{\prime}-\sum_{n \mid q} \sum_{i=1}^{c_{n}}\left[k_{n i} / q_{n}\right] P_{i}^{n},
$$

where $D^{\prime}$ is the projection of $D$ onto $W^{\prime}$, and $[x]$ is the largest integer $\leqq x$. Put $\{x\}=x-[x]$. Let $\mu_{v}$ denote -1 times the degree of the right hand side of (5.11). Assume that $(v, q)=1$. Then $s_{n i} v \neq 0\left(\bmod q_{n}\right)$, hence $\left\{k_{n i} / q_{n}\right\}=$ $\left\{s_{n i} v / q_{n}\right\}-1 / q_{n}$. Therefore 


$$
\begin{aligned}
\mu_{v} & =q^{-1} \operatorname{deg}\left(\operatorname{div}\left(f_{0} d z\right)\right)-\sum_{n, i}\left\{k_{n i} / q_{n}\right\} \\
& =(2 \mathfrak{g}-2) / q-\sum_{n, i}\left\{k_{n i} / q_{n}\right\} \\
& =2 \mathfrak{g}^{\prime}-2+\sum_{n, i}\left(1-\left\{s_{n i} v / q_{n}\right\}\right) \\
& >2 \mathfrak{g}^{\prime}-2 .
\end{aligned}
$$

Thus, by the Riemann-Roch theorem, one has

$$
\lambda_{v}=\mathfrak{g}^{\prime}-1+\sum_{n, i}\left(1-\left\{s_{n i} v / q_{n}\right\}\right) .
$$

Observe that $\sum_{v=1}^{q-1} \chi(v)\left\{s v / q_{n}\right\}=0$ for any $s$ if $n>1$. Therefore by (5.10) we obtain

$$
\operatorname{tr}(R)=-\sqrt{-q} \cdot \sum_{r} \sum_{v=1}^{q-1} \chi(v)\left\{v r^{2} / q\right\}=-\sqrt{-q} \cdot(\varphi(q) / 2) \cdot \sum_{k=1}^{q-1} \chi(k) k / q,
$$

which is the desired result.

\section{$\S 6$. The zeta-function of $A$ over a non-abelian extension of $Q$.}

Let $\Gamma$ be a group of level $N$, and let $A$ be defined for a common eigenfunction $f$ in $S_{2}(\Gamma)$ as in Theorem 1. Then one can naturally ask about (the one-dimensional part of) the zeta-function of $A$ over an arbitrary finite algebraic extension $F$ of $\boldsymbol{Q}$. This problem can easily be settled (always up to finitely many bad Euler factors), if $F$ is abelian over $\boldsymbol{Q}$, as shown in [11, $\S 7.9]$ (see also the discussion below). We shall now show that one can determine the zeta-function for a certain class of non-abelian extensions.

Let us denote by $Z(s, A / F)$ the one-dimensional part of the zeta-function of $A$ over $F$, and by $\zeta_{F}(s)$ the Dedekind zeta-function of $F$. We can write

$$
\begin{aligned}
\zeta_{F}(s) & =\prod_{p} \operatorname{det}\left[1-\Psi_{p} p^{-s}\right]^{-1}, \\
Z(s, A / Q) & =\prod_{p} \operatorname{det}\left[1-\Phi_{p} p^{-s}\right]^{-1} \quad \text { (the product for all good primes) }
\end{aligned}
$$

with complex matrices $\Phi_{p}$ and $\Psi_{p}$ given for each prime $p$; the size of $\Phi_{p}$ (resp. $\Psi_{p}$ ) is $2 \cdot \operatorname{dim}(A)(\operatorname{resp} .[F: \boldsymbol{Q}])$; the matrices are invertible for almost all $p$. Then we see easily that

$$
Z(s, A / F)=\prod_{p} \operatorname{det}\left[1-\left(\Phi_{p} \otimes \Psi_{p}\right) p^{-s}\right]^{-1}
$$

This applies to any abelian variety $A$ defined over $\boldsymbol{Q}$.

Now take a common eigen-function $f(z)=\sum_{n} a_{n} e^{2 \pi i n z}$, with $a_{1}=1$, of all Hecke operators in $S_{k}(\Gamma)$, with any positive integer $k$, and put 


$$
\begin{aligned}
L(s, f)=\sum_{n=1}^{\infty} a_{n} n^{-s} & =\prod_{p}\left(1-a_{p} p^{-s}+\varepsilon(p) p^{k-1-2 s}\right)^{-1} \\
& =\prod_{\boldsymbol{p}} \operatorname{det}\left[1-\Xi_{p} p^{-s}\right]^{-1}
\end{aligned}
$$

with complex matrices $\boldsymbol{E}_{p}$ of size 2. Here $\varepsilon(p)$ is a character of $(\boldsymbol{Z} / N \boldsymbol{Z})^{\times}$ such that

$$
f \mid\left[\left[\begin{array}{ll}
a & b \\
c & d
\end{array}\right]\right]_{k}=\varepsilon(d) f \quad \text { for all }\left[\begin{array}{ll}
a & b \\
c & d
\end{array}\right] \in \Gamma_{0}(N) .
$$

Then we define an Euler product

$$
L(s, f, F)=\prod_{p} \operatorname{det}\left[1-\left(\Xi_{p} \otimes \Psi_{p}\right) p^{-s}\right]^{-1},
$$

which obviously converges in some half plane. The last part of Theorem 1 implies that, if $k=2$ and $A$ corresponds to $f$, then, under the condition stated there, $Z(s, A / F)$ coincides with $\prod_{\sigma \in I} L\left(s, f_{\sigma}, F\right)$, up to finitely many Euler factors. It is natural to conjecture that if $k>1, L(s, f, F)$ can always be continued to a holomorphic function on the whole $s$-plane satisfying a functional equation. (If $k=1$, one will have to allow the function to have finitely many poles.) Thus our purpose is to show that this is so at least for a certain class of extensions $F$ of $\boldsymbol{Q}$. To define it, we consider the following condition on a finite group $G$ :

(X) Every irreducible representation of $G$ by complex matrices is either one-dimensional or induced from a one-dimensional representation of a subgroup of index 2.

It can easily be shown that the following types of groups satisfy the condition: abelian group; dihedral group; generalized quaternion group (i.e., a group of order $4 \mathrm{~m}$ generated by two elements $r$ and $s$ with the relations $\left.r^{2}=s^{m}, s^{2 m}=1, r s r^{-1}=s^{-1}\right)$; a homomorphic image of a group satisfying $(\mathrm{X})$; the product of an abelian group and a group satisfying (X).

Let $E$ be a Galois extension of $\boldsymbol{Q}$ such that $\mathrm{Gal}(E / \boldsymbol{Q})$ satisfies (X), and let $F$ be any subfield of $E$. It is this type of extension $F$, for which we are going to show that $L(s, f, F)$ can be determined. Write simply $G$ for $\operatorname{Gal}(E / \boldsymbol{Q})$, and let $L(s, E / \boldsymbol{Q}, \varphi)$ denote the Artin $L$-function with a character $\varphi$ of $G$. By virtue of $(\mathrm{X}), \zeta_{F}(s)$ can be written as a finite product

$$
\zeta_{F}(s)=\prod_{\mu} L\left(s, E / \boldsymbol{Q}, \varphi_{\mu}\right) \times \prod_{\nu} L\left(s, E / \boldsymbol{Q}, \phi_{\nu}\right),
$$

where $\varphi_{\mu}$ is a one-dimensional character of $G$, and $\phi_{\nu}$ is a character induced from an abelian character of a subgroup $H_{\nu}$ of $G$ of index 2. Each $\varphi_{\mu}$ corresponds to a primitive character $\varphi_{\mu}^{*}$ of $\left(\boldsymbol{Z} / m_{\mu} \boldsymbol{Z}\right)^{\times}$with a positive integer $m_{\mu}$, and 


$$
L\left(s, E / \boldsymbol{Q}, \varphi_{\mu}\right)=\prod_{\boldsymbol{p}}\left(1-\varphi_{\mu}^{*}(p) p^{-s}\right)^{-1} .
$$

As to $\phi_{\nu}$, take the subfield $M_{\nu}$ of $E$ corresponding to $H_{\nu}$. Then $\left[M_{\nu}: \boldsymbol{Q}\right]=2$, and $L\left(s, E / \boldsymbol{Q}, \phi_{\nu}\right)$ coincides with an $L$-function

$$
L\left(s, M_{\nu}, \chi_{\nu}\right)=\prod_{\mathfrak{p}}\left(1-\chi_{\nu}(\mathfrak{p}) N(\mathfrak{p})^{-s}\right)^{-1}
$$

of $M_{\nu}$ with a primitive character $\chi_{\nu}$ of finite order, where the product is extended over all prime ideals $\mathfrak{p}$ of $M_{\nu}$ prime to the conductor of $\chi_{\nu}$. Put

$$
L\left(s, M_{\nu}, \chi_{\nu}\right)=\sum_{n} b_{\nu n} n^{-s}=\prod_{p} \operatorname{det}\left[1-\Psi_{\nu p} p^{-s}\right]^{-1}
$$

with complex numbers $b_{\nu n}$ and complex matrices $\Psi_{\nu p}$ of size 2 . Then

where

$$
L(s, f, F)=\prod_{\mu} L\left(s, f, \varphi_{\mu}^{*}\right) \times \prod_{\nu} L\left(s, f, M_{\nu}, \chi_{\nu}\right),
$$

$$
\begin{gathered}
L\left(s, f, \varphi_{\mu}^{*}\right)=\prod_{p} \operatorname{det}\left[1-\varphi_{\mu}^{*}(p) \Xi_{p} p^{-s}\right]^{-1}=\sum_{n} \varphi_{\mu}^{*}(n) a_{n} n^{-s}, \\
L\left(s, f, M_{\nu}, \chi_{\nu}\right)=\prod_{p} \operatorname{det}\left[1-\left(\Xi_{p} \otimes \Psi_{\nu p}\right) p^{-s}\right]^{-1} .
\end{gathered}
$$

By an easy formal computation, we see that

$$
L\left(s, f, M_{\nu}, \chi_{\nu}\right)=\left(\sum_{n=1}^{\infty} a_{n} b_{\nu n} n^{-s}\right)\left(\sum_{n=1}^{\infty}\left(\frac{D_{\nu}}{n}\right) \chi_{\nu}((n)) \varepsilon(n) n^{k-1-2 s}\right),
$$

where $D_{\nu}$ is the discriminant of $M_{\nu}$. Note that $\operatorname{det}\left(\Psi_{\nu p}\right)=\left(\frac{D_{\nu}}{p}\right) \chi_{\nu}((p))$, and $\operatorname{det}\left(\Xi_{p}\right)=\varepsilon(p) p^{k-1}$. The Dirichlet series $L\left(s, f, \varphi_{\mu}^{*}\right)$ can be continued to a holomorphic function on the whole s-plane satisfying a functional equation, since $\sum_{n} \varphi_{\mu}^{*}(n) a_{n} e^{2 \pi i n z}$ is an element of $S_{k}\left(\Gamma_{1}\left(N_{\mu}\right)\right)$ for some $N_{\mu}$ (see [11, Prop. $3.64])$.

To study the nature of $L\left(s, f, M_{\nu}, \chi_{\nu}\right)$, we first observe that $L\left(s, M_{\nu}, \chi_{\nu}\right)$ is the Mellin transform of an automorphic form on $G L_{2}\left(\boldsymbol{Q}_{A}\right)$. This is essentially due to Hecke and Maass, and included, as a special case, in the results of Jacquet and Langlands [6], and Weil [17]. Especially, if $M_{\nu}$ is imaginary, and if we put $g_{\nu}(z)=\sum_{n} b_{\nu n} e^{2 \pi i n z}$, then $g_{\nu}$ is a modular form of weight 1 satisfying

$$
g_{\nu} \mid\left[\left[\begin{array}{ll}
a & b \\
c & d
\end{array}\right]\right]_{1}=\left(\frac{D_{\nu}}{d}\right) \chi_{\nu}((d)) g_{\nu} \quad \text { for all }\left[\begin{array}{ll}
a & b \\
c & d
\end{array}\right] \in \Gamma_{0}\left(D_{\nu} \cdot N\left(\mathfrak{c}_{\nu}\right)\right),
$$

where $c_{\nu}$ is the conductor of $\chi_{\nu}$. Therefore $L\left(s, f, M_{\nu}, \chi_{\nu}\right)$ is similar to the Dirichlet series considered by Rankin [10]. Recently Jacquet [7] has given a general treatment of Dirichlet series of type (6.1). Thus it is virtually possible to obtain the desired property, or at least something very close to it, of $L\left(s, f, M_{\nu}, \chi_{\nu}\right)$ or of $L(s, f, F)$, though one may have to work out the 
details to get a precise statement.

For example, consider the simplest non-abelian case in which $E$ is a non-abelian extension of $\boldsymbol{Q}$ of degree 6, whose unique quadratic subfield is imaginary, and take $F$ to be a cubic subfield of $E$. Then $\zeta_{F}(s)=\zeta_{\boldsymbol{Q}}(s) L(s, M, \chi)$ with the $L$-function $L(s, M, \chi)$ of $M$ for a character $\chi$ of order 3, corresponding to the extension $E / M$, hence

$$
L(s, f, F)=L(s, f) L(s, f, M, \chi) .
$$

Put $L(s, M, \chi)=\sum_{n} b_{n} n^{-s}, g(z)=\sum_{n} b_{n} e^{2 \pi i n z}$. Then $g$ is a modular form of weight 1 , satisfying a relation of the type (6.2). (In this special case, one has $\chi((d))=1$.) Applying the method of $[14, \S 3]$ to $f$ and $g$, we can show that $L(s, f, M, \chi)$ can be continued to an entire function in $s$, if $k>1$. To obtain the exact form of the functional equation, one has to assume that $f \mid\left[\left[\begin{array}{cr}0 & -1 \\ N & 0\end{array}\right]\right]_{k}$ is a common eigen-function of Hecke operators, and make considerations similar to Ogg [9] and the author $[\mathbf{1 4}, \S 3]$; but we shall not treat this problem further in this paper.

\section{References}

[1] A. O. L. Atkin and J. Lehner, Hecke operators on $\Gamma_{0}(m)$, Math. Ann., 185 (1970), 134-160.

[2] W. Casselman, On some results of Atkin and Lehner, to appear.

[3] W.-L. Chow, On the quotient variety of an abelian variety, Proc. Nat. Acad. Sci. U.S. A., 38 (1952), 1039-1044.

[4] E. Hecke, Über ein Fundamentalproblem aus der Theorie der elliptischen Modulfunktionen, Abh. Math. Sem. Hamburg, 6 (1928), 235-257 (=Math. Werke, 525547).

[5] E. Hecke, Über das Verhalten der Integrale 1. Gattung bei Abbildungen, insbesondere in der Theorie der elliptischen Modulfunktionen, Abh. Math. Sem. Hamburg, 8 (1930), 271-281 (=Math. Werke, 548-558).

[6] H. Jacquet and R. P. Langlands, Automorphic forms on $G L(2)$, Lecture notes in mathematics, 114, Springer, 1970.

[7] H. Jacquet, Automorphic forms on $G L(2)$, Part II, Lecture notes in mathematics, 278, Springer, 1972.

[8] T. Miyake, On automorphic forms on $G L_{2}$ and Hecke operators, Ann. of Math., 94 (1971), 174-189.

[9] A. P. Ogg, On a convolution of $L$-series, Invent. math., 7 (1969), 297-312.

[10] R.A. Rankin, Contributions to the theory of Ramanujan's function $\tau(n)$ and similar arithmetical functions I, II, Proc. Cambridge Phil. Soc., 35 (1939), 351372.

[11] G. Shimura, Introduction to the arithmetic theory of automorphic functions, Publ. Math. Soc. Japan, No. 11, Iwanami Shoten and Princeton University Press, 1971.

[12] G. Shimura, On elliptic curves with complex multiplication as factors of the jacobians of modular function fields, Nagoya Math. J., 43 (1971), 199-208. 
[13] G. Shimura, Class fields over real quadratic fields and Hecke operators, Ann. of Math., 95 (1972), 130-190.

[14] G. Shimura, On modular forms of half integral weight, Ann. of Math, 97 (1973), 440-481.

[15] A. Weil, Variétés abéliennes et courbes algébriques, Hermann, Paris, 1948.

[16] A. Weil, Über die Bestimmung Dirichletscher Reihen durch Funktionalgleichungen, Math. Ann., 168 (1967), 149-156.

[17] A. Weil, Dirichlet series and automorphic forms, Lecture notes in mathematics, 189, Springer, 1971.

Goro SHIMURA

Department of Mathematics

Princeton University

Princeton, New Jersey

U.S. A. 